\title{
Ictiofauna del río Manso (Caldas, Colombia)
}

\section{Ichthyofauna of the Manso River (Caldas, Colombia)}

\author{
Wilton Aguiar Gómez [i], Gian Carlo Sánchez Garcés 누 , Paula Andrea Bonilla (i)
}

\section{Resumen}

Se presenta un listado de los peces de la cuenca media y baja del río Manso (Caldas, Colombia), el cual consolida los resultados de 33 campañas de muestreo que se realizaron en ocho estaciones distribuidas en la zona media y baja del río y una quebrada tributaria, además de la información previamente existente. En total se han registrado para el río Manso 68 especies de peces. En el periodo 2012-2014 se registraron 55 especies, de las cuales 15 están incluidas en el Libro Rojo de Peces de Agua Dulce de Colombia, 12 son especies de importancia para consumo humano y las demás representan interés biológico y ecológico.

Palabras clave. Diversidad. Lista de especies. Magdalena medio. Riqueza íctica.

\begin{abstract}
This paper presents a list of the fishes of the middle and lower part of the Manso River basin, in the department of Caldas, Colombia. It consolidates the results of 33 sampling events at 8 stations distributed in the middle and lower Manso river zone and a tributary stream, in addition to previously available information. A total of 68 species of fish have been recorded for the Manso River. In the period 2012-2014, 55 species were registered, of which 15 are included in the Red Book of Freshwater Fish of Colombia, 12 are species of importance for human consumption and the others that represent biological and ecological interest.
\end{abstract}

Keywords. Diversity. Fish riches. List of species. Middle Magdalena.

\section{Introducción}

La región andina de Colombia es reconocida por su alta diversidad biótica y los grandes procesos de transformación de los ecosistemas naturales originales, a causa del desarrollo incontrolado de actividades productivas (Rodríguez et al., 2004). Por lo anterior, esta región ha sido priorizada por parte de organizaciones nacionales e internacionales para la generación de estrategias que garanticen la conservación (IAvH, 2004) y la implementación de programas de recuperación, especialmente en las regiones cafeteras y ganaderas.

La riqueza de especies de plantas, anfibios, reptiles, aves y mamíferos ha sido bien documentada en esta región (Rodríguez et al., 2004). No obstante, en el caso de los peces, a pesar de haberse realizado algunos esfuerzos (Briñez-Vásquez, 2004; García-Melo, 2005; Maldonado-Ocampo et al., 2005; Lasso et al., 2011), se siguen actualizando los listados para las cuencas del Amazonas, Putumayo, Orinoco, Sinú, Magdalena, Cauca, Atrato y Patía (DoNascimiento et al., 2018).

La ictiofauna andina es importante para los pobladores de la región y para los estudios biogeográficos del Neotrópico, y se encuentra en un alto grado de amenaza debido a los efectos de las actividades humanas y el cambio climático; este último podría generar cambios irreversibles en los ecosistemas de los Andes tropicales, trayendo como resultado la disminución o desaparición de especies (Anderson \& Maldonado-Ocampo, 2011). 
Son pocos los estudios sobre los peces de la cuenca del río Manso. La información para este grupo se encuentra principalmente en informes técnicos realizados a partir de la implementación o seguimiento a planes de manejo u otros, siendo generalmente poco difundida. El trabajo de Restrepo-Santamaría \& Álvarez-León (2011), basado en una revisión bibliográfica, presenta para el Departamento de Caldas un listado de 180 especies, pertenecientes a 31 familias y diez órdenes. En ese listado se señalan parael río Manso 29 especies (el $16.1 \%$ de las especies del departamento), agrupadas en 6 órdenes y 11 familias.

Con base en la información recopilada por la empresa ISAGEN durante monitoreos mensuales entre los años 2012 y 2014, se generó un listado de peces para la cuenca media-baja de río Manso, aportando información sobre la riqueza de especies en este sistema, que se complementó con información secundaria, procurando contribuir a un conocimiento más amplio de la ictiofauna de la región.

\section{Materiales y métodos}

La cuenca del río Manso pertenece a la hoya hidrográfica del río La Miel, que, a su vez, es afluente del río Magdalena (Figura 1), y comparte las características propias de la zona media del río Magdalena, entre ellas el régimen climático bimodal, con dos periodos marcados de lluvias y dos estiajes a lo largo del año (Pareja et al., 2014).

Se realizaron 33 muestreos mensuales entre abril de 2012 y diciembre de 2014, en 7 estaciones ubicadas sobre la cuenca media-baja de río Manso y en una quebrada tributaria del río en la zona baja (Tabla 1, Figura 2).

Como artes de pesca se emplearon un equipo de pesca eléctrica (excepto en la cuenca baja), dos atarrayas con diferentes diámetros de ojo de malla $(2.5 \mathrm{~cm}$ en la cuenca media y $5 \mathrm{~cm}$ en la cuenca baja) y una red de arrastre (chinchorro) de $4 \mathrm{~m}$ de longitud, $1.50 \mathrm{~m}$ de alto y $0.3 \mathrm{~cm}$ de ojo de malla. Para estandarizar las capturas, se recorrieron $50 \mathrm{~m}$ de longitud aguas arriba en cada estación de muestreo, barriendo una de las orillas del cauce. La intensidad del muestreo fue de 20 minutos para la electropesca y el chinchorro, mientras que para la atarraya se realizaron 20 lances por punto de muestreo.

Los peces, en lo posible, identificados hasta nivel de especie. El material fijado fue depositado en el Museo
Departamental de Ciencias Naturales del Instituto para la Investigación y Preservación del Patrimonio Cultural y Natural del Valle del Cauca INCIVA (IMCN), después de su determinación y comparación con el material de referencia. La lista taxonómica se realizó siguiendo la propuesta de DoNascimiento et al. (2017). Los nombres válidos fueron confirmados en el Catálogo de peces de la Academia Californiana de Ciencias (Eschmeyer et al. 2019).

La lista obtenida se complementó con la de RestrepoSantamaría \& Álvarez-León (2011). Sin embargo, para los análisis de riqueza, abundancia y variación temporal se usaron solo los datos obtenidos durante las campañas de muestreo de 2012-2014.

\section{Resultados}

Para el área de estudio se capturaron 6262 individuos, distribuidos en 6 órdenes, 20 familias, 35 géneros y 55 especies (Anexo 1), de las cuales 48 fueron confirmadas y 7 se dejaron a nivel de género. Characiformes y Siluriformes alcanzaron las mayores abundancias, con porcentajes del 48.4 y $44.4 \%$ de los individuos, respectivamente. Los demás órdenes incluyeron menos del $4 \%$ de los ejemplares, siendo Myliobatiformes el de menor representación, con el $0.03 \%$. Siluriformes, con el 47.8 $\%$ de los registros (7 familias, 13 géneros y 26 especies), seguido por Characiformes, con el $36.4 \%$ (8 familias, 15 géneros y 20 especies), fueron los órdenes más diversos, seguidos de Cichliformes (7.3\%), con 3 géneros y 4 especies. Cyprinodontiformes y Gymnotiformes registraron una riqueza del $3.6 \%$ cada uno (Tabla 2).

Las familias Characidae y Loricariidae reportaron la mayor riqueza, seguidas por Trichomycteridae, Astroblepidae y Cichlidae (Figura 3). Numéricamente, Loricariidae. Characidae y Prochilodontidae tuvieron los recuentos más elevados y su contribución general fue del $79.9 \%$. Astroblepidae y Trichomycteridae alcanzaron cuantificaciones del $11.8 \%$, mientras que las demás familias aportaron el porcentaje restante.

A escala temporal, se mantuvo la dominancia numérica de Characidae y Loricariidae a lo largo de los periodos evaluados. Del total de familias, el $47.4 \%$ se reportaron en los tres años de estudio, mientras que el porcentaje restante mostró disminuciones de la abundancia entre el segundo y tercer año, siendo el caso más notorio el de Tichomycteridae, que pasó de 241 a 11 individuos. 

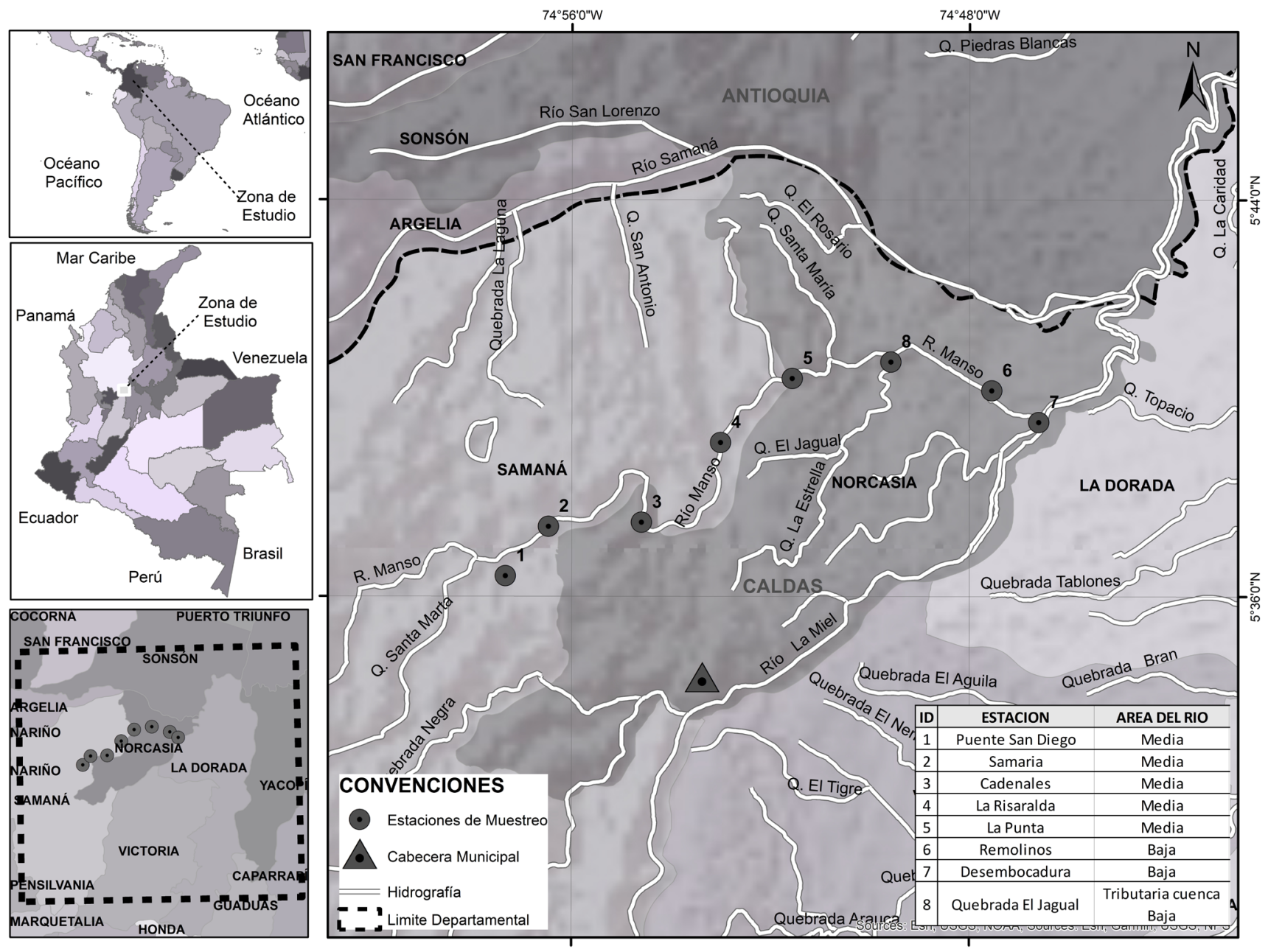

Figura 1. Mapa de la cuenca del río Manso en el departamento de Caldas, Colombia, y sitios donde se realizaron los muestreos de peces.

Tabla 1. Estaciones de muestreo de peces sobre la cuenca del río Manso, Caldas, Colombia.

\begin{tabular}{llllc}
\hline Estación de muestreo & Área del río & Latitud & Longitud & Altitud (m s. n. m.) \\
\hline Puente San Diego & Media & $5^{\circ} 36^{\prime} 24.9^{\prime \prime}$ & $74^{\circ} 57^{\prime} 19.9^{\prime \prime}$ & 653 \\
\hline Samaria & Media & $5^{\circ} 37^{\prime} 23.5^{\prime \prime}$ & $74^{\circ} 36^{\prime} 03.7^{\prime \prime}$ & 610 \\
\hline Cadenales & Media & $5^{\circ} 37^{\prime} 16.3^{\prime \prime}$ & $74^{\circ} 54^{\prime} 18.5^{\prime \prime}$ & 536 \\
\hline La Risaralda & Media & $5^{\circ} 33^{\prime} 35.4^{\prime \prime}$ & $74^{\circ} 52^{\prime} 48.2^{\prime \prime}$ & 411 \\
\hline La Punta & Media & $5^{\circ} 40^{\prime} 21.1^{\prime \prime}$ & $74^{\circ} 50^{\prime} 39.2^{\prime \prime}$ & 243 \\
\hline Remolinos & Baja & $5^{\circ} 40^{\prime} 09.2^{\prime \prime}$ & $74^{\circ} 47^{\prime} 32.9^{\prime \prime}$ & 209 \\
\hline Desembocadura & Baja & $5^{\circ} 39^{\prime} 30.9^{\prime \prime}$ & $74^{\circ} 46^{\prime} 36.2^{\prime \prime}$ & 197 \\
\hline Quebrada El Jagual & Tributaria cuenca Baja & $5^{\circ} 36^{\prime} 28.8^{\prime \prime}$ & $74^{\circ} 57^{\prime} 22.0^{\prime \prime}$ & 217 \\
\hline
\end{tabular}



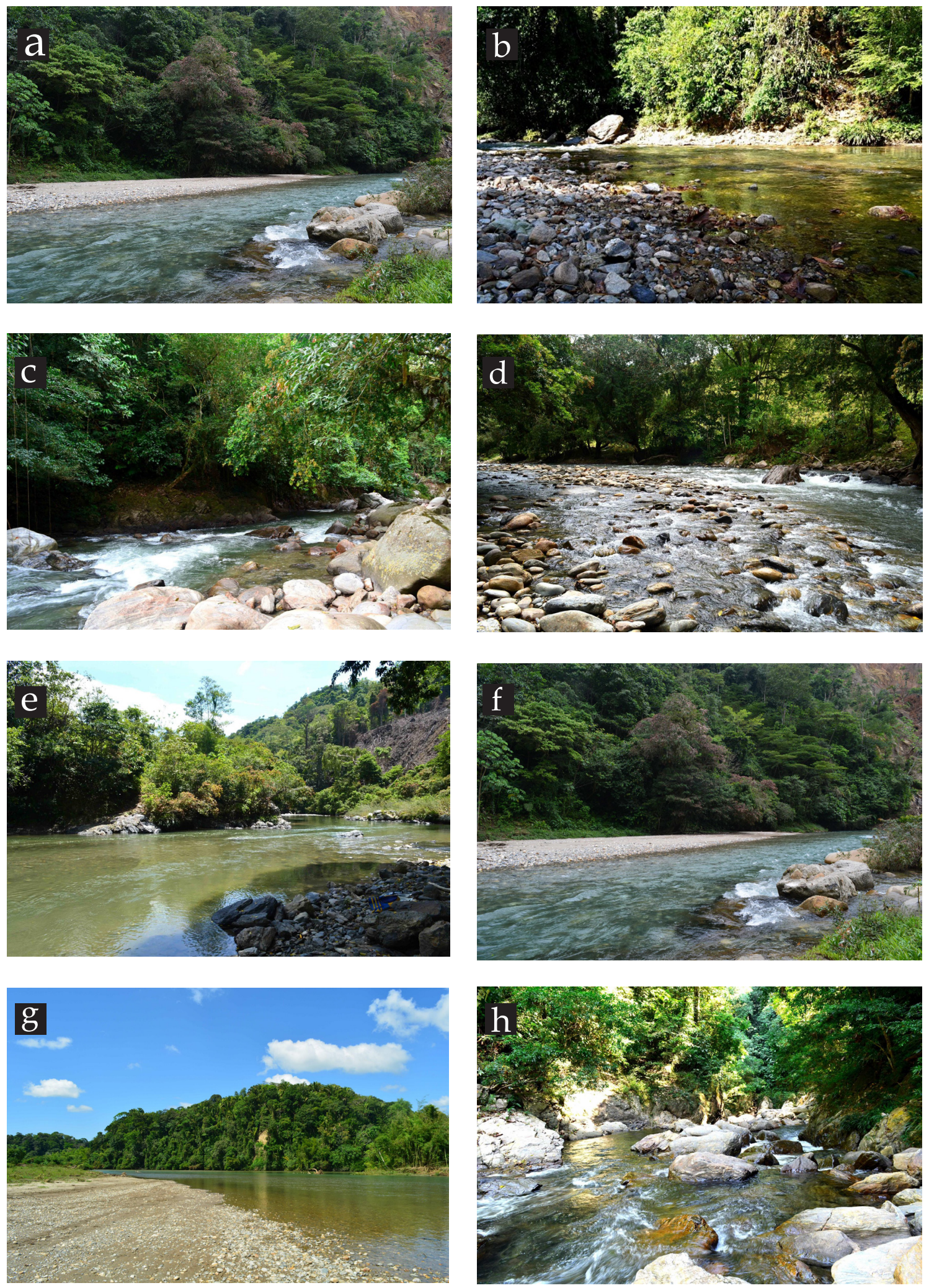

Figura 2. Estaciones donde se realizaron los muestreos de peces en la cuenca del río Manso, Caldas, Colombia. a. Puente San Diego; b. Samaria; c. Cadenales; d. La Risaralda; e. La Punta; f. Remolinos; g. Desembocadura; h. Quebrada El Jagual. 
Tabla 2. Número de familias, géneros y especies peces presentes en la cuenca media y baja del río Manso, Caldas, Colombia.

\begin{tabular}{lllllll}
\hline Orden & $\begin{array}{l}\text { Número de } \\
\text { familias }\end{array}$ & $\begin{array}{l}\text { Pocentaje(\%) } \\
\text { de familias }\end{array}$ & $\begin{array}{l}\text { Número de } \\
\text { géneros }\end{array}$ & $\begin{array}{l}\text { Pocentaje (\%) } \\
\text { de géneros }\end{array}$ & $\begin{array}{l}\text { Número de } \\
\text { especies }\end{array}$ & $\begin{array}{l}\text { Pocentaje (\%) } \\
\text { de especies }\end{array}$ \\
\hline Myliobatiformes & 1 & 5 & 1 & 2.9 & 1 & 1.8 \\
\hline Characiformes & 8 & 40 & 15 & 42.9 & 20 & 36.4 \\
\hline Siluriformes & 7 & 35 & 13 & 37.1 & 26 & 47.3 \\
\hline Gymnotiformes & 1 & 5 & 1 & 2.9 & 2 & 3.6 \\
\hline Cyprinodontiformes & 2 & 10 & 2 & 5.7 & 2 & 3.6 \\
\hline Cichliformes & 1 & 5 & 3 & 8.6 & 4 & 7.3 \\
\hline
\end{tabular}

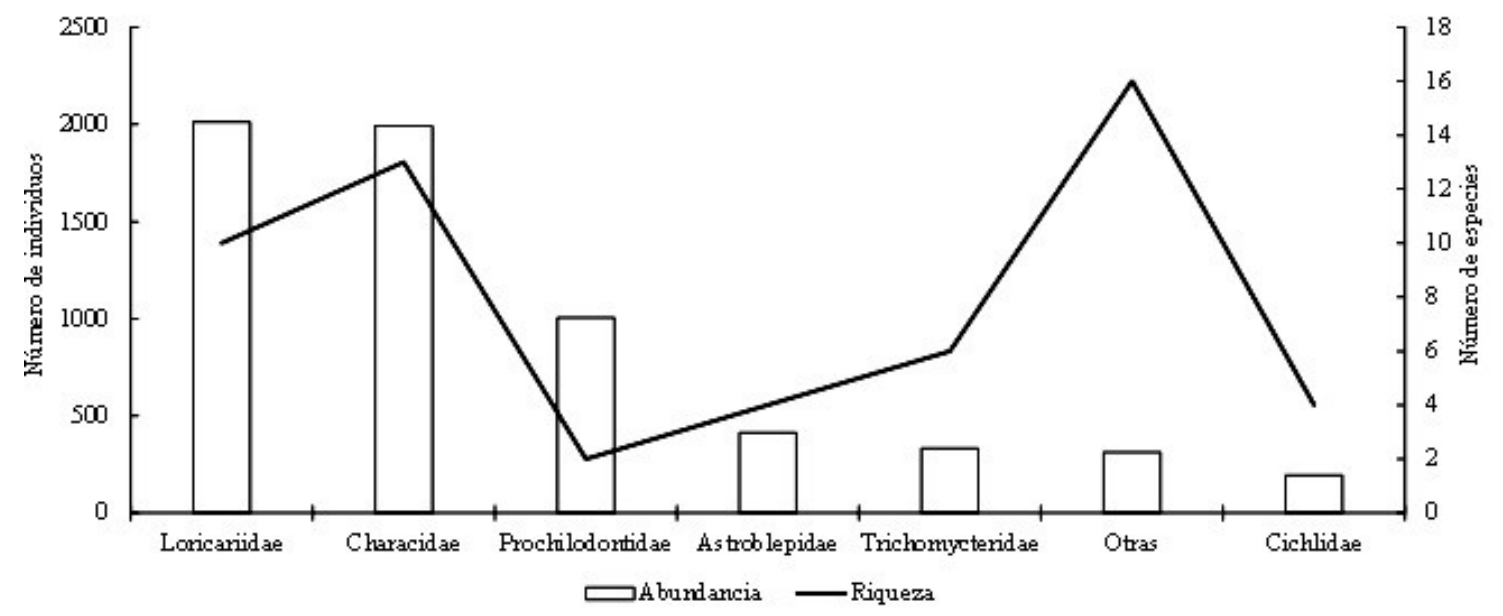

Figura 3. Abundancia y riqueza de familias de peces en la cuenca media y baja de río Manso, Caldas, Colombia.

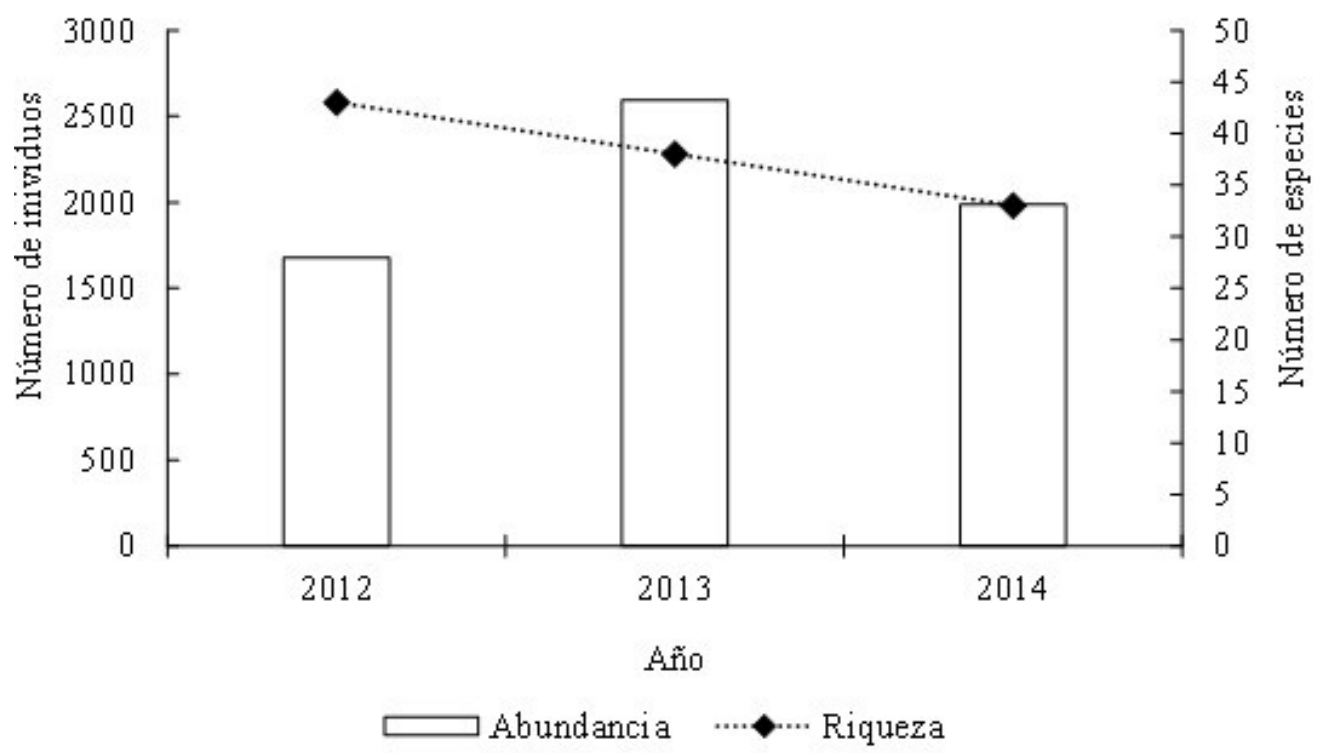

Figura 4. Riqueza y abundancia de peces en tres años de muestreo en la cuenca media y baja de río Manso, Caldas, Colombia. 
El número de especies registradas fue decreciendo consecutivamente, encontrándose el máximo valor en el año 2012, cuando se identificaron 43 especies (Figura 4). Para los dos años restantes se presentaron disminuciones en la riqueza del 16.3 y $23.3 \%$ respectivamente, con respecto a esta cifra. El $42 \%$ de las especies encontradas en los inventarios de 2012 y 2013 no se registraron para 2014 y, en dicho año el número de nuevos registros se incrementó en un 9.1 \% (Saccodon dariensis, Creagrutus brevipinnis, Saccoderma hastatus, Cetopsis othonops y Caquetaia umbrifera).

Las especies más abundantes fueron Chaetostoma fisheri (24.8 \%), Prochilodus magdalenae (15.4\%), Creagrutus magdalenae (10.1\%), Hemibrycon raqueliae (7.8 \%) y Astroblepus homodon (6.2\%), que representaron más del 60 $\%$ de las capturas. Tres especies (C. fisheri, C. magdalenae y $H$. raqueliae) estuvieron ampliamente distribuidas entre las diferentes estaciones durante los tres años, y una especie (C. fisheri) estuvo en todas las estaciones durante el 2012, y en el 75 \% de ellas en el 2013 y 2014.

Ocho especies estuvieron ampliamente distribuidas entre las diferentes estaciones durante el primer año: Chaetostoma fisheri estuvo en el $100 \%$, Creagrutus affinis, Astroblepus homodon y Chaetostoma sp., 75 \%, mientras que Creagrutus magdalenae, Hemibrycon raqueliae, Trichomycterus retropinnis y Chaetostoma milesi estuvieron en el $62.5 \%$ de las estaciones. Durante el segundo año fueron nueve las especies que estuvieron ampliamente distribuidas: C. magdalenae, 100 \%, Hemibrycon caucanus, H. raqueliae, Trichomycterus banneaui, T. retropinnis, A. homodon, C. fisheri y Geophagus steindachneri, $75 \%$, y Astroblepus grixalvii, $62.5 \%$. Finalmente, en el 2014 hubo una disminución en el número de especies de amplia distribución: $H$. raqueliae, A. homodon y C. fisheri, 75 $\%$, y H. caucanus y Chaetostoma sp. $62.5 \%$.

Una de las especies (Icthyoelephas longirostris) está categorizada como En Peligro, nueve como Vulnerables (Curimata mivartii, Brycon moorei, Salminus affinis, Prochilodus magdalenae, Megaleporinus muyscorum, Characidium phoxocephalum, Pimelodus grosskopfii, Sorubim cuspicaudus y Apteronotus magdalenensis) y cuatro como Casi Amenazadas (Potamotrygon magdalenae, Brycon rubricauda, Hypostomus hondae y Kronoheros umbrifer; Mojica \& lasso, 2012). Se registraron 28 especies endémicas de la cuenca del Magdalena (DoNacimiento et al., 2017) (51 \%) y 10 migratorias, seis de las cuales hacen migraciones medianas, que consisten en desplazamientos entre 100 y $500 \mathrm{~km}$ (Zapata \& Usma, 2013).
Desde el punto de vista del valor de los recursos pesqueros, se registraron 16 especies que son reconocidas en la zona como de interés pesquero (Lasso et al., 2011) y de éstas, 13 se reconocen como especies de pesca para consumo y las tres restantes tienen valor como especies ornamentales.

\section{Discusión}

La riqueza de especies del río La Miel, del cual es tributario el río Manso, representa un poco más del 50 \% del número de especies reportado para la cuenca del río Magdalena-Cauca y del 7 \% de la riqueza del país (Maldonado-Ocampo et al., 2008; Jiménez-Segura et al., 2014a).

El presente listado de especies del río Manso corresponde casi a la cuarta parte de la riqueza reportada para la cuenca Magdalena-Cauca (DoNascimiento et al., 2017) y cerca de la mitad de los registros de peces del río La Miel (López-Casas et al., 2014), con especies de diversos patrones ecomorfológicos, además de un número considerable de especies endémicas. Sin embargo, trabajos anteriores reportan para el río Manso 54 especies (Restrepo-Santamaría \& Álvarez-León, 2011), de las cuales 21 no se hallaron en el presente estudio, 32 fueron comunes entre ambos trabajos y 17 se reportan como nuevos registros (Anexo 1), aumentando el número de especies para la cuenca a 68 , número que podría ascender con la determinación de los ejemplares que faltan por confirmar.

La predominancia de especies de los órdenes Characiformes y Siluriformes coincide con lo encontrado los Andes tropicales (Anderson \& Maldonado-Ocampo, 2011) y en el valle medio del río Magdalena. Estos dos grupos han experimentado la radiación adaptativa más importante (85\% de la riqueza), en los sistemas fluviales de Suramérica (Albert \& Reis, 2011).

Por su parte, la dominancia de las familias Loricariidae y Characidae se ajusta al patrón encontrado en el norte de Suramérica donde estos grupos contribuyen con cerca del 51 \% de la riqueza de especies (Albert \& Reis, 2011), un patrón que es constante para la mayoría de los ríos andinos (Jaramillo-Villa et al., 2010; Anderson \& Maldonado-Ocampo, 2011; Carvajal-Quintero et al., 2015). En la cuenca del Magdalena, en particular, estas familias comprenden 65 y 31 especies, respectivamente (DoNascimiento et al., 2017). 
La predominancia de los Siluriformes en las cuencas del departamento de Caldas se debe principalmente a las familias Astroblepidae, Loricariidae y Trichomycteridae.

Estas familias presentan especies que se han especializado y adaptado a variados ambientes de zonas bajas, como en el caso de los loricáridos, y a zonas de mayor altura, como los astroblépidos y algunos tricomictéridos (Villa-Navarro et al., 2005; Anderson \& MaldonadoOcampo, 2011). Para el caso de los loricáridos, su abundancia en sectores como San Diego, Samaria, Cadenales y La Risaralda estaría relacionada con las corrientes fuertes, los sustratos pedregosos y las aguas claras y bien oxigenadas.

Hacia el tramo medio del río Manso predominaron especies de pequeño porte, siendo importantes los carácidos Hemibrycon caucanus, H. raqueliae y Creagrutus magdalenae, los cuales estarían asociados a cauces pequeños, de ancho menor a $5 \mathrm{~m}$, con corriente entre moderada y rápida, con sustratos conformados por rocas, gravas, arenas y lodos, y bordeados por vegetación ribereña que aporta sombra (Jiménez-Segura et al., 2014b).

Las especies que viven en las áreas ritrales se alimentan de material alóctono como hojas, semillas e insectos que caen al agua, a diferencia de las especies que se distribuyen hacia la desembocadura (áreas potamales), las cuales obtienen de la materia orgánica y el detritus su principal fuente de alimento (Lowe McConnell, 1999). Lo anterior permitiría entender la abundancia de individuos de la familia Characidae, que en general se caracterizan por sus hábitos omnívoros. Por otro lado, las familias Astroblepidae (Astroblepus homodon) y Trichomyteridae (Trichomycterus banneaui), con dietas basadas en insectos acuáticos, se encontraron asociadas a fondos arenosos con guijarros y rocas, vegetación ribereña, material alóctono y perifiton, con profundidades de 0.10-0.90 m, flujos rápidos y moderados, y márgenes abruptas y excavadas.

Characidium phoxocephalum fue el único representante de la familia Crenuchidae y solo se registró para el sector de Samaria, donde tuvo una baja abundancia $(0.18$ $\%$ ). Pese a su pequeño tamaño, las especies del género Characidium que presentan hábitos bentónicos se consideran sensibles a la degradación del hábitat, particularmente por sedimentación (Rodríguez-Olarte, 2011). En la cuenca del río Manso esta especie se encuentra asociada a cauces con buena cobertura vegetal, fondos rocosos con presencia de fango y con formación esporádica de playas.
Hacia la cuenca media se destacan especies exclusivas del sector de Cadenales, donde se registraron Saccodon dariensis, Brycon moorei, B. rubricauda, Chaetostoma sp., Lasiancistrus caucanus y Cynodonichthys magdalenae, las cuales se asocian a aguas transparentes de corriente suave, en cauces con sustratos donde dominan rocas $y$ arena.

En el tramo bajo de la cuenca, en la zona de influencia del río La Miel, se registraron especies como Prochilodus magdalenae, Salminus affinis, Brycon moorei, Potamotrygon magdalenae, Curimata mivartii, Hypostomus hondae, Dasyloricaria filamentosa, Pimelodus blochii, Pimelodus grosskopfii, Creagrutus magdalenae, Ctenolucius hujeta, Sorubim cuspicaudus y Megaleporinus muyscorum las cuales suelen ser más frecuentes por debajo de los 200 msnm. Algunas de estas especies son migratorias y presentan importancia dentro de la pesca artesanal y de subsistencia, además de considerarse como determinantes para el área de conservación Medio MagdalenaBarranca-Honda (Lasso et al., 2017).

Dentro de los peces migratorios, los más abundantes y frecuentes fueron los integrantes de la familia Prochilodontidae, considerados como importante componente de la pesca comercial y de subsistencia en los sistemas de agua dulce de Suramérica (Jiménez-Segura et al. 2014b). En la cuenca del río Manso dicha familia estuvo representada por Ichthyoelephas longirostris y Prochilodus magdalenae, que realizan migraciones medianas que se caracterizan por desplazamientos entre los 100 y 500 km (Zapata Padilla \& Usma Oviedo, 2013). Ambas especies se distribuyeron exclusivamente en las localidades de Remolinos y La Desembocadura, que son los sectores de mayor influencia del río La Miel, el cual ha sido identificado como ruta migratoria y donde se presentan movimientos ascendentes, principalmente en periodo de aguas bajas (Jiménez et al., 2014b).

En el río La Miel, en la zona de desembocadura del río Manso, la ictiofauna se caracterizó por la riqueza de especies típicas de las zonas por debajo de 200 m s.n.m. Muchas de ellas son migrantes estacionales (Jiménez-Segura et al., 2014b), en las cuales se da un desplazamiento masivo de peces que buscan condiciones apropiadas para desovar, constituyendo los ríos Manso y Samaná áreas de desove (Moreno et al., 2013). Las migraciones les a los organismos enfrentarse a la heterogeneidad ambiental, tanto temporal como espacial, de una manera previsible y resulta en la presencia sucesiva de individuos de una misma especie en diferentes hábitats (Usma et al., 2013). 
De igual forma, las sardinas Argopleura magdalenensis y Astyanax fasciatus están referenciadas principalmente para la zona baja, ya que habitan en ríos con cauces de mayor tamaño, donde se distribuyen en sitios protegidos de la corriente. La ocurrencia de $A$. fasciatus en los sectores de Remolinos y La Desembocadura concuerda con lo mencionado por Jiménez-Segura et al. (2014a), quienes indican que esta sardina prefiere los hábitats por debajo de $500 \mathrm{~m}$. La amplia distribución de los carácidos, tanto en las zonas ritrales como potamales, evidencia que se trata de especies generalistas, cuyas características ecomorfológicas les permiten el movimiento a lo largo de la columna de agua, así como el uso de diferentes tipos de hábitats (Conde-Saldaña et al., 2017).

La cuenca del río Manso es vulnerable al crecimiento poblacional, así como a procesos de deforestación, contaminación, ganadería extensiva, agricultura, extracción de material, y sobrepesca, factores que están incidiendo en la alta proporción de especies amenazadas (18.1\%) (Tognelli et al., 2016).

Algunas especies de los géneros Astroblepus, Chaetostoma, Hemibrycon y Trichomycterus requieren la revisión por parte de especialistas, ya que existe la posibilidad de que se trate de nuevas especies. Adicionalmente, se recomienda hacer estudios que permitan generar estrategias de conservación, teniendo en cuenta que en la región existen proyectos de desarrollo que podrían incidir en los ecosistemas acuáticos.

\section{Referencias}

Albert, J. S. \& Reis, R. E. (Eds) (2011). Historical Biogeography of Neotropical Freshwater Fishes. Berkeley, CA: University of California Press. https://doi.org/10.1525/california/9780520268685.001.0001

Anderson. E. P. \& Maldonado-Ocampo. J. A. (2011). A regional perspective on the diversity and conservation of tropical andean fishes. Conservation Biology, 25(1), 3039. https:// doi.org/10.1111/j.1523-1739.2010.01568.x

Briñez-Vásquez G. N. (2004). Distribución altitudinal. diversidad y algunos aspectos ecológicos de la familia Astroblepidae (Pisses: Siluriformes) en la cuenca del río Coello (Tolima). (Trabajo de Grado). Ibagué, Colombia: Programa de Biología, Facultad de Ciencias. Universidad del Tolima. $134 \mathrm{pp}$.
Carvajal-Quintero. J. D.. Escobar. F.. Jaramillo-Villa. U. \& Maldonado-Ocampo. J. A. (2015). Variation in freshwater fish assemblages along a regional elevation gradient in the northern Andes. Colombia. Ecology and Evolution, 5(13), 2608-2620. https:// doi.org/10.1002/ ece3.1539

Conde-Saldaña. C. C., Albornoz-Garzón. J. G., López-Delgado. E. O. \& Villa-Navarro. F. (2017). Ecomorphological relationships of fish assemblages in a tras-Andean drainage. Upper Magdalena River Basin. Colombia. Neotropical Ichthyology, 15(4), 54-61. https:// doi.org/10.1590/1982-0224-20170037

DoNascimiento. C,. Herrera-Collazos. E. E., Herrera G. A., Ortega-Lara. A., Villa-Navarro. F. A., UsmaOviedo. J. S. \& Maldonado-Ocampo. J. A. (2017). Checklist of freshwater fishes of Colombia: a Darwin core alternative to the updating problem. ZooKeys, 708 (25), 25-138. https:// doi.org/10.3897/ zookeys.708.13897

DoNascimiento, C., Herrera Collazos, E. E. \& MaldonadoOcampo, J, A. (2018). Lista de especies de peces de agua dulce de Colombia / Checklist of the freshwater fishes of Colombia. v2.10. Asociación Colombiana de Ictiólgos. Dataset/Checklist. http://doi.org/10.15472/ numrso

Eschmeyer. W. N., Fricke. R. \& van der Laan. R. (2019). Catalog of fishes. California Academy of Sciences, 3882(1), 1-230.

García-Melo L. J. (2005). Distribución. diversidad y ecología básica de la familia Trichomycteridae (Ostariophysi: Siluriformes). en la cuenca del río Coello. departamento del Tolima. (Trabajo de Grado). Ibagué, Colombia: Programa de Biología, Facultad de Ciencias. Universidad del Tolima. $149 \mathrm{pp}$.

IAvH. (2004). Informe anual (2003). Proyecto Conservación y Uso Sostenible de la Biodiversidad de los Andes Colombianos. Bogotá D.C.: Instituto de Investigación de Recursos Biológicos Alexander von Humboldt.

IGAC. (1990). Caldas. Aspectos geográficos. Bogotá D.C.: IGAC.

Jaramillo-Villa, U., Maldonado-Ocampo, J. A. \& Escobar, F. (2010). Altitudinal variation in fish assemblage diversity in streams of the central Andes of Colombia. Journal of Fish Biology, 76, 2401-2417. https:/ / doi.org/10.1111/j.1095-8649.2010.02629.x

Jiménez-Segura. L. F, Restrepo-Santamaría, D., López-Casas, S., Delgado, J., Valderrama, M., Álvarez, J. \& Gomez, D. (2014a). Ictiofauna y desarrollo del sector hidroeléctrico en la cuenca del río MagdalenaCauca. Colombia. Biota Colombiana, 15(2), 3-25. 
Jiménez-Segura, L. F., Maldonado-Ocampo, J. A. \& Pérez-Gallego, C. M. (2014b). Gradiente de recuperación longitudinal en la estructura de la ictiofauna en un río andino regulado. Biota Colombiana, 15(2), 61-80.

Lasso, C. A., Gutiérrez, F de P., Morales-Betancourt, M. A., Agudelo Córdoba, E., Ramírez-Gil, H. \& AjiacoMartínez, R. E. (Eds.) (2011). Pesquerías continentales de Colombia: cuencas del Magdalena-Cauca. Sinú. Canalete. Atrato. Orinoco. Amazonas y vertiente del Pacífico. Serie Editorial Recursos Hidrobiológicos y Pesqueros Continentales de Colombia. Bogotá D.C.: Instituto de Investigación de los Recursos Biológicos Alexander von Humboldt. 304 pp.

Lasso, C. A., Córdoba, D. \& Morales-Betancourt, M.A. (Eds.) (2011). XVI. Áreas clave para la conservación de la biodiversidad dulceacuícola amenazada en Colombia: moluscos. cangrejos. peces. tortugas. cocodrílidos. aves y mamíferos. Serie Editorial Recursos Hidrobiológicos y Pesqueros Continentales de Colombia. Bogotá D.C.: Instituto de Investigación de los Recursos Biológicos Alexander von Humboldt. 353 pp.

López-Casas, S., L. F. Jiménez-Segura, F. Villa-Navarro, C. Pérez, D. M. Gualtero-Leal \& Angel-Rojas. V. J. (2013). Peces potádromos migratorios en la cuenca del río Magdalena. Pp. 52. En: Colombia. Programa y resúmenes del XII Congreso Colombiano de Ictiología y III Encuentro Suramericano de Ictiólogos. Bogotá D.C.: Pontificia Universidad Javeriana, ACICTIOS.

Lowe-Mcconnell, R. (1999). Estudios ecológicos de comunidades de peixes tropicais. São Paulo: EDUSP. 534 pp.

Maldonado-Ocampo, J.A., Ortega-Lara, A., Usma O., J.S., Galvis, V. G., Villa Navarro, F.A.,Vásquez, L., Prada- Pedreros,S. \& Ardila R. C. (2005). Peces de los Andes de Colombia. Bogotá D.C.: Instituto de Investigación de Recursos Biológicos Alexander von Humboldt. 346 pp.

Mojica, J. I. \& Lasso, Á. L. (2012). Libro rojo de peces dulceacuícolas de Colombia. Bogotá D.C.: Instituto de Investigación de Recursos Biológicos Alexander von Humboldt. 319 pp.

Moreno, C., Jiménez-Segura. L. F. \& Pérez. C. (2013). Reproducción de peces migratorios en un sistema regulado: el caso del río La Miel. Pp. 124. En: Colombia. 2013. Programa y resúmenes del XII Congreso Colombiano de Ictiología y III Encuentro Suramericano de Ictiólogos. Bogotá D.C.: Pontificia Universidad Javeriana, ACICTIOS.
Pareja-Carmona, M. I., Jiménez-Segura, L. F. \& Ochoa-orrego, L. E. (2014). Variación espacio-temporal de las larvas de tres especies de peces migratorios en el cauce del río Magdalena (Colombia). durante el ciclo hidrológico 2006-2007. Actualidades Biológicas, 36, 33-38.

Reis, R., Kullander, E. S. \& Ferraris, Jr. C. (2003). Check List of the Freshwater Fishes of South and Central America. Porto Alegre: Pontifícia Universidade Católica do Rio Grande do Sul. Museu de Ciências e Tecnologia.

Restrepo-Santamaría, D. \& Álvarez-León, R. (2011). Peces del departamento de Caldas. Colombia. Biota Colombiana, 12(1), 117-135.

Rodríguez, N., Armenteras, D., Morales, M. \& Romero, M. (2004). Ecosistemas de los Andes colombianos. Bogotá D.C.: Instituto de Investigación de Recursos Biológicos Alexander von Humboldt. 154 pp.

Rodríguez-Olarte, J.J. \& Taphorn, D. (2011). Northern South America. Magdalena and Maracaibo basins. Historical biogeography of Neotropical freshwater fishes, 243-184.

https://doi.org/10.1525/california/9780520268685.003.0015 Tognelli, M.F., Lasso, C.A., Bota-Sierra, C.A., JiménezSegura, L.F. \& Cox, N.A. (Eds). (2016). Estado de Conservación y Distribución de la Biodiversidad de Agua Dulce en los Andes Tropicales. Gland. Suiza. Cambridge. UK y Arlington. USA: UICN. xii + 199 pp. https://doi.org/10.2305/IUCN.CH.2016.02.en

Usma J. S., Villa-Navarro. F., Lasso. C. A., Castro. F., Zúñiga-Upegui. P. T., Cipamocha. C., OrtegaLara. A., Ajiaco. R. E., Ramírez-Gil. H., Jiménez. L. F., MaldonadoOcampo. J. A., Muñoz. J. \& Suárez. J. T. (2013). Peces dulceacuícolas de Colombia. Pp. 79-128. En: Zapata. L. A. \& Usma. J. S. (Eds.). Guía de las especies migratorias de la biodiversidad en Colombia. Peces. Vol. 2. Bogotá D.C.: Ministerio de Ambiente y Desarrollo Sostenible / WWFColombia. 486 pp.

Villa-Navarro, F. A., Briñez-Vasquez, L. J., GarcíaMelo, J. E., Reinoso-Flores, G. \& Esquivel, H. E. (2005). Biodiversidad de la cuenca de los ríos Prado y Amoyá. (Trabajo de Grado). Ibagué. Colombia: Universidad del Tolima. $150 \mathrm{pp}$.

Zapata Padilla, L. A. \& Usma Oviedo, J. S. (2013). Guía de las especies Migratorias de la Biodiversidad en Colombia. Peces. Vol. 2. Bogotá D.C.: Ministerio de Ambiente y Desarrollo Sostenible / WWF-Colombia. 486 pp. 
Anexo 1. Listado taxonómico de los peces de la cuenca media y baja del río Manso, Caldas, Colombia. Especies reportadas por Restrepo-Santamaría \& Álvarez-León (2011) (R\&A); especies que coinciden con el presente estudio (ü); especies que no se encontraron en el presente estudio $\left(^{*}\right)$; nuevos registros para la cuenca (à); especies registradas en 2012 (X); especies registradas en $2013(€)$ y especies registradas en $2014(Y)$.

\begin{tabular}{|c|c|c|c|c|c|c|c|c|c|c|c|c|c|}
\hline Taxa & $\begin{array}{l}\text { Colección } \\
\text { de } \\
\text { referencia }\end{array}$ & E1 & E2 & E3 & E4 & E5 & E6 & E7 & E8 & 2012 & 2013 & 2014 & Total \\
\hline \multicolumn{14}{|l|}{ MYLIOBATIFORMES } \\
\hline \multicolumn{14}{|l|}{ Potamotrygonidae } \\
\hline Potamotrygon magdalenae & - & & & & & & & $x €$ & & 1 & 1 & & 2 \\
\hline \multicolumn{14}{|l|}{ CHARACIFORMES } \\
\hline \multicolumn{14}{|l|}{ Crenuchidae } \\
\hline $\begin{array}{l}\text { Characidium } \\
\text { phoxocephalum }\end{array}$ & 8493 & & $X €$ & & & & & & & 1 & 1 & & 2 \\
\hline \multicolumn{14}{|l|}{ Parodontidae } \\
\hline Saccodon dariensis & 8498 & & & $¥$ & & & & & & & & 1 & 1 \\
\hline \multicolumn{14}{|l|}{ Anostomidae } \\
\hline Megaleporinus muyscorum & - & & & & & & $X € Y$ & $X € \Psi$ & & 16 & 20 & 7 & 43 \\
\hline \multicolumn{14}{|l|}{ Curimatidae } \\
\hline Curimata mivartii & - & & & & & & & $€$ & & & 2 & & 2 \\
\hline \multicolumn{14}{|l|}{ Prochilodontidae } \\
\hline Icthyoelephas longirostris & - & & & & & & $X €$ & $X € ¥$ & & 17 & 24 & 2 & 43 \\
\hline Prochilodus magdalenae & - & & & & & & $X € ¥$ & $X € ¥$ & & 305 & 225 & 432 & 962 \\
\hline \multicolumn{14}{|l|}{ Ctenoluciidae } \\
\hline Ctenolucius hujeta & 8491 & & & & & & & $€$ & & & 1 & & 1 \\
\hline \multicolumn{14}{|l|}{ Characidae } \\
\hline Saccoderma hastata & 8521 & & & & & & $¥$ & & & & & 2 & 2 \\
\hline Gephyrocharax melanocheir & 8522 & & & & & $x$ & $¥$ & $x$ & & 2 & & 5 & 7 \\
\hline Hemibrycon caucanus & 8523 & X€¥ & $X € ¥$ & $X € ¥$ & $€ ¥$ & $X ¥$ & $€$ & & & 45 & 100 & 139 & 284 \\
\hline Hemibrycon raqueliae & 8524 & $X € \Psi$ & X€¥ & $X € ¥$ & $X € ¥$ & X€¥ & $€$ & & $¥$ & 128 & 228 & 134 & 490 \\
\hline Hemibrycon sp. & 8525 & & $x$ & $x$ & $x$ & $x$ & & & & 23 & & & 23 \\
\hline Creagrutus affinis & 8526 & $x$ & $x$ & & $x$ & $x$ & $X ¥$ & $X ¥$ & & 105 & & 36 & 141 \\
\hline Creagrutus magdalenae & 8527 & X€¥ & $X €$ & $€$ & $€$ & $X €$ & $X € ¥$ & $X € ¥$ & $€$ & 105 & 374 & 155 & 634 \\
\hline Argopleura magdalenensis & 8528 & $x$ & $€$ & & & $X € ¥$ & $X \in \Psi$ & $X € ¥$ & & 122 & 203 & 30 & 355 \\
\hline Astyanax fasciatus gr. & 8505 & & & & & & $X € ¥$ & $x$ & & 16 & 1 & 6 & 23 \\
\hline \multicolumn{14}{|l|}{ Bryconidae } \\
\hline Brycon moorei & - & & & $X €$ & & & $X €$ & $X ¥$ & & 6 & 4 & 2 & 12 \\
\hline Brycon rubricauda & - & & & $x$ & & & & & & 1 & & & \\
\hline
\end{tabular}




\begin{tabular}{|c|c|c|c|c|c|c|c|c|c|c|c|c|c|}
\hline Salminus affinis & - & & & & & & $X$ & $X € ¥$ & & 4 & 2 & 2 & 8 \\
\hline \multicolumn{14}{|l|}{ GYMNOTIFORMES } \\
\hline \multicolumn{14}{|l|}{ Apteronotidae } \\
\hline Apteronotus eschmeyeri & - & & $x$ & & & & & & $X$ & 2 & & & 2 \\
\hline Apteronotus magdalenensis & - & & & & & & $x$ & & & 1 & & & 1 \\
\hline \multicolumn{14}{|l|}{ SILURIFORMES } \\
\hline \multicolumn{14}{|l|}{ Trichomycteridae } \\
\hline Trichomycterus banneaui & 8516 & $X € Y$ & $€ ¥$ & $€$ & $€ ¥$ & $X € ¥$ & & & $€$ & 11 & 59 & 6 & 76 \\
\hline Trichomycterus retropinnis & 8515 & $X €$ & $X € Y$ & $X € \Psi$ & $€$ & $X €$ & $X €$ & & & 55 & 142 & 5 & 202 \\
\hline Trichomycterus sp. & 8517 & $€$ & $€$ & $€$ & & $€$ & & & & & 13 & & 13 \\
\hline Trichomycterus sp 1 & 8518 & & $X$ & & & & & & & 1 & & & 1 \\
\hline Trichomycterus sp 2 & 8519 & & & $x$ & & & & & & 3 & & & 3 \\
\hline Trichomycterus sp 3 & 8520 & & $x$ & & & $x$ & & & & 4 & & & 4 \\
\hline Paravandellia phaneronema & 8497 & & $€$ & $€$ & & & $X €$ & & & 1 & 27 & & 28 \\
\hline \multicolumn{14}{|l|}{ Astroblepidae } \\
\hline Astroblepus grixalvii & 8507 & $x$ & $€ ¥$ & $€$ & & $€$ & & & $€$ & 1 & 9 & 1 & 11 \\
\hline Astroblepus guentheri & 8509 & & $x$ & & $X$ & & & & & 7 & & & 7 \\
\hline Astroblepus homodon & 8506 & X€¥ & $X € ¥$ & $X € ¥$ & $X € \Psi$ & $X € ¥$ & & & $X € ¥$ & 135 & 142 & 112 & 389 \\
\hline Astroblepus sp. & 8508 & & $X$ & $X$ & $x$ & & & & & 6 & & & 6 \\
\hline \multicolumn{14}{|l|}{ Loricariidae } \\
\hline $\begin{array}{l}\text { Sturisomatichthys pana- } \\
\text { mensis }\end{array}$ & 8496 & & & & & & $€$ & & & & 4 & & 4 \\
\hline Crossoloricaria variegata & 8495 & & & & & & $X €$ & $X € ¥$ & & 5 & 16 & 4 & 25 \\
\hline Dasyloricaria filamentosa & 8494 & & & & & & $€$ & $€ ¥$ & & & 8 & 3 & 11 \\
\hline Chaetostoma fisheri & $14 / 12 / 8511$ & $X € \Psi$ & $X € \Psi$ & $X € ¥$ & X€¥ & $X € \Psi$ & $X Y$ & $X Y$ & X€Y & 266 & 707 & 582 & 1555 \\
\hline Chaetostoma leucomelas & - & & & $€$ & $€$ & & $€$ & & $€$ & & 6 & & 6 \\
\hline Chaetostoma milesi & - & & $X \in \Psi$ & $X € \Psi$ & $X €$ & & $x$ & & $X € \Psi$ & 50 & 56 & 87 & 193 \\
\hline Chaetostoma thomsoni & - & & $x$ & $€ ¥$ & & & $x$ & & $€$ & 7 & 3 & 1 & 11 \\
\hline Chaetostoma sp. & 8513 & $X ¥$ & $X ¥$ & X€ & $x$ & $X ¥$ & & & $X ¥$ & 172 & 3 & 14 & 189 \\
\hline Hypostomus hondae & - & & & & & & $€$ & $X ¥$ & & 3 & 4 & 1 & 8 \\
\hline Lasiancistrus caucanus & - & & & $€ ¥$ & & & & & & & 2 & 1 & 3 \\
\hline \multicolumn{14}{|l|}{ Cetopsidadae } \\
\hline Cetopsis othonops & 8504 & & & & & & & & $¥$ & & & 3 & 3 \\
\hline \multicolumn{14}{|l|}{ Aspredinidae } \\
\hline Dupouyichthys sapito & 8503 & & & & & & $X €$ & $X €$ & & 2 & 13 & & 15 \\
\hline
\end{tabular}




\begin{tabular}{|c|c|c|c|c|c|c|c|c|c|c|c|}
\hline Heptapteridae & & & & & & & & & & & \\
\hline Pimelodella chagresi & 8492 & & & & $X € ¥$ & $€$ & $€$ & 3 & 5 & 3 & 11 \\
\hline \multicolumn{12}{|l|}{ Pimelodidae } \\
\hline Pimelodus blochii & - & & & & & & $x$ & 1 & & & 1 \\
\hline Pimelodus grosskopfii & - & & & & & & $x$ & 1 & & & 1 \\
\hline Sorubim cuspicaudus & - & & & & & $x$ & $X €$ & 4 & 2 & & 6 \\
\hline \multicolumn{12}{|l|}{ CICHLIFORMES } \\
\hline Geophagus steindachneri & 8501 & $€$ & $€ \Psi$ & $€ ¥$ & $X € \Psi$ & $X €$ & $X €$ & 23 & 77 & 69 & 169 \\
\hline Andinoacara latifrons & 8502 & & & & $x$ & & $€$ & 1 & 2 & & 3 \\
\hline Caquetaia kraussii & - & $€ ¥$ & & & $€ ¥$ & $x$ & & 1 & 4 & 8 & 13 \\
\hline Kronoheros umbrifer & - & & & & & & & & & & \\
\hline CYPRINODONTIFORMES & & & & & & & $¥$ & & & 1 & 1 \\
\hline \multicolumn{12}{|l|}{ Cynolebiidae } \\
\hline $\begin{array}{l}\text { Cynodonichthys } \\
\text { magdalenae }\end{array}$ & 8499 & & $€ ¥$ & & & & & & 83 & 60 & 143 \\
\hline \multicolumn{12}{|l|}{ Poeciliidae } \\
\hline Poecilia caucana & 8500 & & $€ ¥$ & & & $X €$ & $X € ¥$ & 14 & 22 & 73 & 109 \\
\hline
\end{tabular}


Wilton Aguiar Gómez

Universidad de Caldas

Manizalez, Colombia

wiltonaguiargomez@hotmail.com

https://orcid.org/0000-0003-4674-6273

\section{Gian Carlo Sánchez Garcés}

Fundación para la Investigación y el Desarrollo Sostenible

Cali, Colombia

hiyuxa@hotmail.com

https://orcid.org/0000-0002-6494-0881

\section{Ictiofauna del río Manso (Caldas, Colombia)}

Citación del artículo: Aguiar, W., Sánchez-Garcés, G.C \& Bonilla, P.A. (2020). Ictiofauna del río Manso (Caldas, Colombia). Biota Colombiana, 21(1), 28-40. DOI: 10.21068/ c2020.v21n01a03.

\section{Paula Andrea Bonilla}

Fundación para la Investigación y el Desarrollo Sostenible Cali, Colombia

paula2177@hotmail.com

https://orcid.org/0000-0003-2214-8164

Recepción: 27 de febrero de 2019

Aprobado: 10 de diciembre de 2019

40 | Biota Colombiana 21 (1) - 2020 